\title{
Blind Source Separation Coping with the Change of the Number of Sources
}

\author{
Masanori Ito $^{1}$, Noboru Ohnishi ${ }^{1}$, Ali Mansour ${ }^{2}$, and Mitsuru Kawamoto ${ }^{3,4}$ \\ ${ }^{1}$ Graduate School of Information Science, Nagoya University \\ Furo-cho, Chikusa-ku, Nagoya,464-8603 Japan \\ ito-m@nagoya-u.jp \\ ${ }^{2}$ Lab. $\mathrm{E}^{3} \mathrm{I}^{2}$, ENSIETA, 29806 Brest cedex 09, France \\ ${ }^{3}$ Advanced Industrial Science and Technology, \\ Central 2, 1-1-1 Umezono, Tsukuba, Ibaraki, 305-8568 Japan \\ ${ }^{4}$ Bio-mimetic Control Research Center, RIKEN, \\ 2271-120 Anagahora, Shimoshidami, Moriyama-ku, Nagoya, 463-0003 Japan
}

\begin{abstract}
This manuscript deals with the blind source separation problem with an instantaneous but dynamical mixture model. This study is limited to the case when the number of sources is time-variant. Theoretically, when new sources are detected, a new separating matrix should be estimated in order to extract all sources. However this effort implies an overwhelm computational cost. Our idea consists to use the previous separating matrix which was estimated before the appearance of the new sources. Owing to this point, the computational time and cost can be effectively reduced compared with the conventional separation scheme. Our new algorithm was corroborated with many simulations. Some results are given in the manuscript. The obtained and presented results clearly show that the proposed method outperformed the conventional method in processing time as well as in separation quality.
\end{abstract}

Keywords: blind source separation, time-variant system, dynamical instantaneous mixtures, independent component analysis.

\section{Introduction}

In the last decade, independent component analysis (ICA) has been greatly developed 12 1 . ICA is often used to solve blind source separation (BSS) problems, which means the estimation of original sources from their mixtures, using the only observed signals without any knowledge about the sources and the mixing process. Applying ICA algorithms the original sources can be estimated up to scaling and permutation factors.

A number of researchers have been struggling with BSS problems. However, most of the researches on BSS assume the time-invariant systems. The number of sources is a very important factor for BSS problems. However, most of the algorithms assumes that the number of sources is constant and known a priori. Ye et al. have proposed the BSS without knowing the number of sources [3]. But they

M. Ishikawa et al. (Eds.): ICONIP 2007, Part II, LNCS 4985, pp. 509-518. 2008.

(C) Springer-Verlag Berlin Heidelberg 2008 


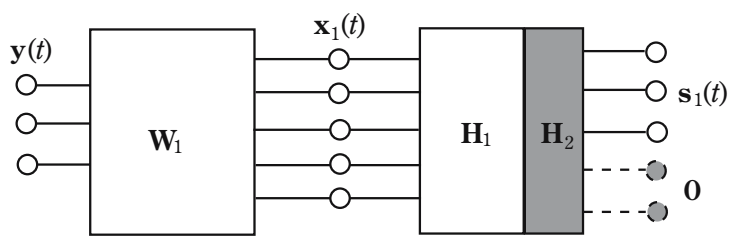

(a)

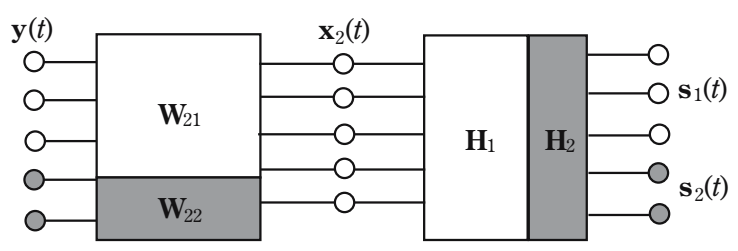

(b)

Fig. 1. Mixing and separating process. (a) The process before the appearance of $s_{2}(t)$. Only $s_{1}(t)$ exists. (b) The process after the appearance of $s_{2}(t)$.

have not considered the change of the number. For practical use, we need a solution of the BSS problems in time-variant systems, where the properties of the mixing process and sources may change. Some researchers have proposed methods to solve the blind separation of moving sources [4.5]. When source moves, the property of the mixing process varies, but the dimensions of the mixing matrix and the sources are constant. Therefore we deal with the case where the number of sources is not constant, that is, the dimensions of the mixing matrix and the sources are variable. Until now, such a problem has not been solved. In particular we consider the case that the number of sources increases because a decrease of sources does not affect the subsequent separation process.

In this manuscript, in order to solve the BSS problem with the change of the number of sources, the following conditions are assumed;

A1) The mixing process is invertible and instantaneous.

A2) There are no noise.

A3) The number of active sources is always known.

A4) The signals are real-valued.

Hereafter, the scheme of the separation coping with the case that new sources appear is proposed. Making use of the covariance matrix of the mixed signals, a new matrix which blocks undesired sources can be estimated. Through computer simulations, it is clarified that the proposed method can work efficiently.

\section{Problem Formulation}

It is supposed that $n_{1}$ sources initially exist for $t<T_{0}$ and at time $t=T_{0}$ new $n_{2}$ sources suddenly appear, i.e., $\left(n_{1}+n_{2}\right)$ sources are active for the period $t \geq T_{0}$. 
The former sources are denoted as $\boldsymbol{s}_{1}(t)$, the latter source $\boldsymbol{s}_{2}(t)$. We call $\boldsymbol{s}_{1}(t)$ initial sources and $\boldsymbol{s}_{2}(t)$ additional sources. We unite them into $\boldsymbol{s}(t)$ :

$$
\boldsymbol{s}(t)=\left[\begin{array}{l}
\boldsymbol{s}_{1}(t) \\
\boldsymbol{s}_{2}(t)
\end{array}\right] \text {. }
$$

Their mixtures are observed by multiple sensors whose number $m$ is greater than or equal to that of whole sources $\left(n_{1}+n_{2}\right)$. Mixed signals are denoted by $\boldsymbol{x}_{1}(t)$ $\left(t<T_{0}\right)$ or $\boldsymbol{x}_{2}(t)\left(t \geq T_{0}\right)$. The initial mixed signals $\boldsymbol{x}_{1}(t)$ can be expressed as follows (See Fig. 1 (a).):

$$
\boldsymbol{x}_{1}(t)=\left[\begin{array}{ll}
\boldsymbol{H}_{1} & \boldsymbol{H}_{2}
\end{array}\right]\left[\begin{array}{c}
\boldsymbol{s}_{1}(t) \\
\mathbf{0}_{n_{2}}
\end{array}\right] .
$$

$\boldsymbol{x}_{1}(t)$ can be rewritten in another way:

$$
\boldsymbol{x}_{1}(t)=\left[\begin{array}{ll}
\boldsymbol{H}_{1} & \boldsymbol{O}_{m \times n_{2}}
\end{array}\right]\left[\begin{array}{l}
\boldsymbol{s}_{1}(t) \\
\boldsymbol{s}_{2}(t)
\end{array}\right],
$$

where $\boldsymbol{H}_{1}$ and $\boldsymbol{H}_{2}$ are $m \times n_{1}$ and $m \times n_{2}$ mixing matrices corresponding to sources $\boldsymbol{s}_{1}(t)$ and $\boldsymbol{s}_{2}(t)$, respectively. Matrices $\boldsymbol{H}_{1}$ and $\left[\boldsymbol{H}_{1} \boldsymbol{H}_{2}\right]$ are assumed to be column full rank. $\mathbf{0}$ and $\boldsymbol{O}$ are a zero vector and a zero matrix, and the subscript shows their dimensions. The above two equations are mathematically equivalent but physically different. In the former way it is supposed that additional sources emit no signals, while in the latter way, additional sources have no paths to sensors. In this research, the latter case of Eq. (3) is considered because of mathematical convenience. In order to achieve BSS for $\boldsymbol{x}_{1}(t)$, an $n_{1} \times m$ separating matrix $\boldsymbol{W}_{1}$ is estimated and the separated signals $\boldsymbol{y}_{1}(t)$ is written as

$$
\boldsymbol{y}_{1}(t)=\boldsymbol{W}_{1} \boldsymbol{x}_{1}(t)=\boldsymbol{P} \boldsymbol{D} \boldsymbol{s}_{1}(t),
$$

where $\boldsymbol{P}$ is a permutation matrix and $\boldsymbol{D}$ is a diagonal matrix which results in a scaling factor. However, if $\hat{\boldsymbol{s}}_{1}(t)=\boldsymbol{P} \boldsymbol{D} \boldsymbol{s}_{1}(t)$ are considered as original sources, permutation and scaling ambiguity can be ignored.

After the appearance of new sources as shown in Fig. 1(b), the mixed signals $\boldsymbol{x}_{2}(t)$ are written as follows:

$$
\boldsymbol{x}_{2}(t)=\left[\begin{array}{ll}
\boldsymbol{H}_{1} & \boldsymbol{H}_{2}
\end{array}\right]\left[\begin{array}{l}
\boldsymbol{s}_{1}(t) \\
\boldsymbol{s}_{2}(t)
\end{array}\right] .
$$

Then a new $\left(n_{1}+n_{2}\right) \times m$ matrix $\boldsymbol{W}_{2}=\left[\begin{array}{l}\boldsymbol{W}_{21} \\ \boldsymbol{W}_{22}\end{array}\right]$ should be estimated. The goal of the BSS problem is to obtain the original sources $\boldsymbol{s}_{1}(t)$ and $\boldsymbol{s}_{2}(t)$ by estimating a separating matrix using ICA.

\section{Separation Scheme}

\subsection{Separation of Initial Sources}

In the first stage, we estimate a separating matrix for initially existing sources applying ICA. Without loss of generality, let us define an ideal matrix $\boldsymbol{W}_{1}$ which is represented without permutation and scaling ambiguity as follows: 


$$
\boldsymbol{W}_{1}=\boldsymbol{H}_{1}^{\dagger}=\left(\boldsymbol{H}_{1}^{T} \boldsymbol{H}_{1}\right)^{-1} \boldsymbol{H}_{1}^{T},
$$

where $^{\dagger}$ denotes the pseudo inverse (Moore-Penrose generalized inverse) operation.

Because $\boldsymbol{H}_{1}$ is unknown, $\boldsymbol{W}_{1}$ should be estimated by applying some independent component analysis (ICA) algorithm to the mixed signal $\boldsymbol{x}_{1}(t)$. Inversely using estimated $\boldsymbol{W}_{1}, \boldsymbol{H}_{1}$ can also be calculated as

$$
\boldsymbol{H}_{1}=\boldsymbol{W}_{1}^{\dagger}=\boldsymbol{W}_{1}^{T}\left(\boldsymbol{W}_{1} \boldsymbol{W}_{1}^{T}\right)^{-1} .
$$

In this stage, we assume that we can estimate an ideal separating matrix, otherwise in the subsequent stage our method cannot avoid to fail the separation. Using the proposed method, the separation error in this stage affects the performance in the subsequent stage.

\subsection{Separation After Sources Addition}

When the appearance of the additional sources is detected, a new separating matrix should be calculated. As well as $\boldsymbol{W}_{1}$ in (6), we can represent an ideal separating matrix $\boldsymbol{W}_{2}=\left[\begin{array}{l}\boldsymbol{W}_{21} \\ \boldsymbol{W}_{22}\end{array}\right]$ as a function of $\boldsymbol{H}_{1}$ and $\boldsymbol{H}_{2}$ :

$$
\begin{aligned}
\boldsymbol{W}_{2}= & {\left[\boldsymbol{H}_{1} \boldsymbol{H}_{2}\right]^{\dagger} } \\
= & {\left[\begin{array}{c}
\boldsymbol{H}_{1}^{\dagger}-\boldsymbol{H}_{1}^{\dagger} \boldsymbol{H}_{2}\left(\boldsymbol{H}_{2}^{T}\left(\boldsymbol{I}_{m}-\boldsymbol{H}_{1} \boldsymbol{H}_{1}^{\dagger}\right) \boldsymbol{H}_{2}\right)^{-1} \boldsymbol{H}_{2}^{T}\left(\boldsymbol{I}_{m}-\boldsymbol{H}_{1} \boldsymbol{H}_{1}^{\dagger}\right) \\
\left(\boldsymbol{H}_{2}^{T}\left(\boldsymbol{I}_{m}-\boldsymbol{H}_{1} \boldsymbol{H}_{1}^{\dagger}\right) \boldsymbol{H}_{2}\right)^{-1} \boldsymbol{H}_{2}^{T}\left(\boldsymbol{I}_{m}-\boldsymbol{H}_{1} \boldsymbol{H}_{1}^{\dagger}\right)
\end{array}\right], }
\end{aligned}
$$

where $\boldsymbol{I}$ denotes an identity matrix with a subscript of the dimension. Note that the matrix $\left(\boldsymbol{I}_{m}-\boldsymbol{H}_{1} \boldsymbol{H}_{1}^{\dagger}\right)$ is symmetric and idempotent, that is, $\left(\boldsymbol{I}_{m}-\boldsymbol{H}_{1} \boldsymbol{H}_{1}^{\dagger}\right)$ is the orthogonal projection [6]. In the rest of this paper, $\left(\boldsymbol{I}_{m}-\boldsymbol{H}_{1} \boldsymbol{H}_{1}^{\dagger}\right)$ is substituted with $\boldsymbol{P}_{1}$. Then the above equation can be rewritten in short form:

$$
\begin{aligned}
\boldsymbol{W}_{2}=\left[\begin{array}{c}
\boldsymbol{W}_{21} \\
\boldsymbol{W}_{22}
\end{array}\right] & =\left[\begin{array}{c}
\boldsymbol{H}_{1}^{\dagger}\left(\boldsymbol{I}_{m}-\boldsymbol{H}_{2}\left\{\boldsymbol{P}_{1} \boldsymbol{H}_{2}\right\}^{\dagger}\right) \\
\left\{\boldsymbol{P}_{1} \boldsymbol{H}_{2}\right\}^{\dagger}
\end{array}\right] \\
& =\left[\begin{array}{c}
\boldsymbol{H}_{1}^{\dagger}\left(\boldsymbol{I}_{m}-\boldsymbol{H}_{2} \boldsymbol{W}_{22}\right) \\
\left\{\boldsymbol{P}_{1} \boldsymbol{H}_{2}\right\}^{\dagger}
\end{array}\right] .
\end{aligned}
$$

$\boldsymbol{W}_{2}$ is also obtained by applying ICA to the mixed signal $\boldsymbol{x}_{2}(t)$. However, we may suffer a computational load, because we have to estimate a big separating matrix of size $\left(n_{1}+n_{2}\right) \times m$. The essence of our method is to reuse the estimated mixing matrix $\boldsymbol{H}_{1}$ and to reduce the cost. Here, let us define a global matrix $\boldsymbol{G}=\left[\begin{array}{l}\boldsymbol{W}_{21} \\ \boldsymbol{W}_{22}\end{array}\right]\left[\begin{array}{ll}\boldsymbol{H}_{1} & \boldsymbol{H}_{2}\end{array}\right]$, which is shown in Fig. 2], If the submatrices of the global matrix satisfy the following conditions:

$$
\begin{aligned}
& \boldsymbol{W}_{21} \boldsymbol{H}_{1}=\boldsymbol{I}_{n_{1}}, \\
& \boldsymbol{W}_{21} \boldsymbol{H}_{2}=\boldsymbol{O}_{n_{1} \times n_{2}}, \\
& \boldsymbol{W}_{22} \boldsymbol{H}_{1}=\boldsymbol{O}_{n_{2} \times n_{1}},
\end{aligned}
$$




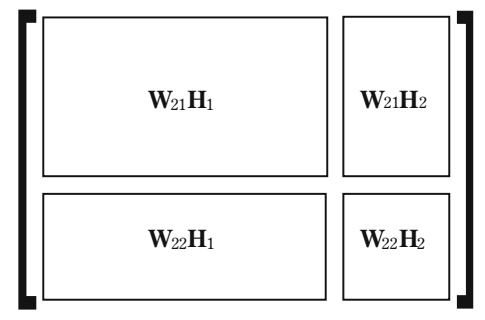

Fig. 2. Global matrix after the new sources appearance

the separation problem can be simplified, because we only have to solve the separation of the part $\boldsymbol{W}_{22} \boldsymbol{H}_{2}$. The proposed scheme to estimate $\boldsymbol{W}_{2}$ satisfying the above conditions is shown below.

Given $\boldsymbol{H}_{2}, \boldsymbol{W}_{2}$ in (8) can be estimated because $\boldsymbol{H}_{1}$ is known. However, there is no information about $\boldsymbol{H}_{2}$. Here, we substitute an arbitrary nonzero matrix $\boldsymbol{A}_{2}$ for $\boldsymbol{H}_{2}$ in $\boldsymbol{W}_{2}$ of Eq. (8) and we obtain

$$
\tilde{\boldsymbol{W}}_{2}=\left[\begin{array}{c}
\tilde{\boldsymbol{W}}_{21} \\
\tilde{\boldsymbol{W}}_{22}
\end{array}\right]=\left[\begin{array}{c}
\boldsymbol{H}_{1}^{\dagger}-\boldsymbol{H}_{1}^{\dagger} \boldsymbol{A}_{2}\left(\boldsymbol{A}_{2}^{T} \boldsymbol{P}_{1} \boldsymbol{A}_{2}\right)^{-1} \boldsymbol{A}_{2}^{T} \boldsymbol{P}_{1} \\
\left(\boldsymbol{A}_{2}^{T} \boldsymbol{P}_{1} \boldsymbol{A}_{2}\right)^{-1} \boldsymbol{A}_{2}^{T} \boldsymbol{P}_{1}
\end{array}\right]
$$

Theorem 1. For any matrix $\boldsymbol{A}_{2}$, the following equation is satisfied,

$$
\tilde{\boldsymbol{W}}_{22} \boldsymbol{H}_{1}=\boldsymbol{O}_{n_{2} \times n_{1}} .
$$

$\tilde{\boldsymbol{W}}_{22}$ works as a blocker [7] of $\boldsymbol{H}_{1}$ even if the mixing matrix $\boldsymbol{H}_{2}$ is unknown. Proof.

$$
\begin{aligned}
\boldsymbol{P}_{1} \boldsymbol{H}_{1} & =\left(\boldsymbol{I}_{m}-\boldsymbol{H}_{1} \boldsymbol{H}_{1}^{\dagger}\right) \boldsymbol{H}_{1} \\
& =\boldsymbol{H}_{1}-\boldsymbol{H}_{1} \boldsymbol{H}_{1}^{\dagger} \boldsymbol{H}_{1} \\
& =\boldsymbol{H}_{1}-\boldsymbol{H}_{1} \\
& =\boldsymbol{O}_{m \times n_{1}} .
\end{aligned}
$$

Therefore $\tilde{\boldsymbol{W}}_{22} \boldsymbol{H}_{1}=\left(\boldsymbol{A}_{2}^{T} \boldsymbol{P}_{1} \boldsymbol{A}_{2}\right)^{-1} \boldsymbol{A}_{2}^{T} \boldsymbol{P}_{1} \boldsymbol{H}_{1}=\boldsymbol{O}_{n_{2} \times n_{1}}$.

Using Theorem 1, it is clear that $\tilde{\boldsymbol{W}}_{21} \boldsymbol{H}_{1}=\boldsymbol{I}_{n_{1}}$.

Hereafter the method to estimated a separating matrix $\hat{\boldsymbol{W}}_{21}$, which satisfies $\hat{\boldsymbol{W}}_{21} \boldsymbol{H}_{2}=\boldsymbol{O}_{n_{1} \times M 2}$, is shown. For the purpose, the following equation is calculated:

$$
\tilde{\boldsymbol{A}}_{2}=\boldsymbol{C}_{x_{2}} \tilde{\boldsymbol{W}}_{22}^{T},
$$

where $\boldsymbol{C}_{x_{2}}$ is the covariance matrix of $\boldsymbol{x}_{2}(t)$. The covariance matrix can be rewritten as 


$$
\boldsymbol{C}_{x_{2}}=\left[\begin{array}{ll}
\boldsymbol{H}_{1} & \boldsymbol{H}_{2}
\end{array}\right] \boldsymbol{C}_{s}\left[\begin{array}{l}
\boldsymbol{H}_{1}^{T} \\
\boldsymbol{H}_{2}^{T}
\end{array}\right],
$$

where $C_{s}$ is the covariance matrix of $s(t)$. Putting Eq. (18) into Eq. (17), we obtain

$$
\begin{aligned}
\tilde{\boldsymbol{A}}_{2} & =\left[\begin{array}{ll}
\boldsymbol{H}_{1} & \boldsymbol{H}_{2}
\end{array}\right] \boldsymbol{C}_{s}\left[\begin{array}{l}
\boldsymbol{H}_{1}^{T} \\
\boldsymbol{H}_{2}^{T}
\end{array}\right] \tilde{\boldsymbol{W}}_{22}^{T} \\
& =\left[\begin{array}{ll}
\boldsymbol{H}_{1} & \boldsymbol{H}_{2}
\end{array}\right]\left[\begin{array}{ll}
\boldsymbol{C}_{s_{1}} & \boldsymbol{O}_{n_{1} \times n_{2}} \\
\boldsymbol{O}_{n_{2} \times n_{1}} & \boldsymbol{C}_{s_{2}}
\end{array}\right]\left[\begin{array}{c}
\boldsymbol{O}_{n_{1} \times n_{2}} \\
\boldsymbol{H}_{2}^{T} \tilde{\boldsymbol{W}}_{22}^{T}
\end{array}\right] \\
& =\boldsymbol{H}_{2} \boldsymbol{C}_{s_{2}} \boldsymbol{H}_{2}^{T} \tilde{\boldsymbol{W}}_{22}^{T},
\end{aligned}
$$

where $\boldsymbol{C}_{s_{1}}$ and $\boldsymbol{C}_{s_{2}}$ are the covariance matrices of $\boldsymbol{s}_{1}(t)$ and $\boldsymbol{s}_{2}(t)$, respectively. For simplicity we replace $\boldsymbol{C}_{s_{2}} \boldsymbol{H}_{2}^{T} \tilde{\boldsymbol{W}}_{22}^{T}$ by $\boldsymbol{M}$. If $\operatorname{det} \boldsymbol{M} \neq 0$, that is, $\operatorname{det} \tilde{\boldsymbol{W}}_{22} \boldsymbol{H}_{2} \neq 0$, we substitute $\tilde{\boldsymbol{A}}_{2}=\boldsymbol{H}_{2} \boldsymbol{M}$ for $\boldsymbol{H}_{2}$ of Eq. (9) to get $\hat{\boldsymbol{W}}_{2}=$ $\left[\begin{array}{l}\hat{W}_{21} \\ \hat{\boldsymbol{W}}_{22}\end{array}\right]$

$$
\hat{\boldsymbol{W}}_{2}=\left[\begin{array}{c}
\boldsymbol{H}_{1}^{\dagger}\left(\boldsymbol{I}_{m}-\boldsymbol{H}_{2} \boldsymbol{M}\left\{\boldsymbol{P}_{1} \boldsymbol{H}_{2} \boldsymbol{M}\right\}^{\dagger}\right) \\
\left\{\boldsymbol{P}_{1} \boldsymbol{H}_{2} \boldsymbol{M}\right\}^{\dagger}
\end{array}\right] .
$$

Here, we notice the following attractive property.

Lemma 1. $\left\{\boldsymbol{P}_{1} \boldsymbol{H}_{2} \boldsymbol{M}\right\}^{\dagger}$ is a generalized inverse [8] of $\boldsymbol{H}_{2} \boldsymbol{M}$.

Proof. A generalized inverse of a matrix $\boldsymbol{B}$, which is denoted as $\boldsymbol{B}^{-}$, satisfies $\boldsymbol{B} \boldsymbol{B}^{-} \boldsymbol{B}=\boldsymbol{B}$. Thus $\boldsymbol{H}_{2} \boldsymbol{M}\left\{\boldsymbol{P}_{1} \boldsymbol{H}_{2} \boldsymbol{M}\right\}^{\dagger} \boldsymbol{H}_{2} \boldsymbol{M}$ is calculated as follows:

$$
\begin{aligned}
& \boldsymbol{H}_{2} \boldsymbol{M}\left\{\boldsymbol{P}_{1} \boldsymbol{H}_{2} \boldsymbol{M}\right\}^{\dagger} \boldsymbol{H}_{2} \boldsymbol{M} \\
& =\boldsymbol{H}_{2} \boldsymbol{M}\left\{\boldsymbol{M}^{T} \boldsymbol{H}_{2}^{T} \boldsymbol{P}_{1} \boldsymbol{H}_{2} \boldsymbol{M}\right\}^{-1} \boldsymbol{M}^{T} \boldsymbol{H}_{2}^{T} \boldsymbol{P}_{1} \boldsymbol{H}_{2} \boldsymbol{M} \\
& =\boldsymbol{H}_{2} \boldsymbol{M} .
\end{aligned}
$$

Therefore $\left\{\boldsymbol{P}_{1} \boldsymbol{H}_{2} \boldsymbol{M}\right\}^{\dagger}$ is a generalized inverse of $\boldsymbol{H}_{2} \boldsymbol{M}$.

Then we have the following theorem.

Theorem 2. $\hat{\boldsymbol{W}}_{21}$ works as a blocker of $\boldsymbol{H}_{1}$, i.e., $\hat{\boldsymbol{W}}_{21} \boldsymbol{H}_{1}=\boldsymbol{O}_{n_{1} \times n_{2}}$.

Proof. Using Lemma 1, it is clear that $\hat{\boldsymbol{W}}_{21}$ is a blocker of $\boldsymbol{H}_{2}$;

$$
\begin{aligned}
\hat{\boldsymbol{W}}_{21} \boldsymbol{H}_{2} & =\boldsymbol{H}_{1}^{\dagger}\left(\boldsymbol{I}_{m}-\boldsymbol{H}_{2} \boldsymbol{M}\left\{\boldsymbol{P}_{1} \boldsymbol{H}_{2} \boldsymbol{M}\right\}^{\dagger}\right) \boldsymbol{H}_{2} \\
& =\boldsymbol{H}_{1}^{\dagger}\left(\boldsymbol{H}_{2}-\boldsymbol{H}_{2} \boldsymbol{M}\left\{\boldsymbol{P}_{1} \boldsymbol{H}_{2} \boldsymbol{M}\right\}^{\dagger} \boldsymbol{H}_{2} \boldsymbol{M} \boldsymbol{M}^{-1}\right) \\
& =\boldsymbol{H}_{1}^{\dagger}\left(\boldsymbol{H}_{2}-\boldsymbol{H}_{2} \boldsymbol{M} \boldsymbol{M}^{-1}\right) \\
& =\boldsymbol{O}_{n_{1} \times n_{2}} .
\end{aligned}
$$


Table 1. Summary of the proposed method

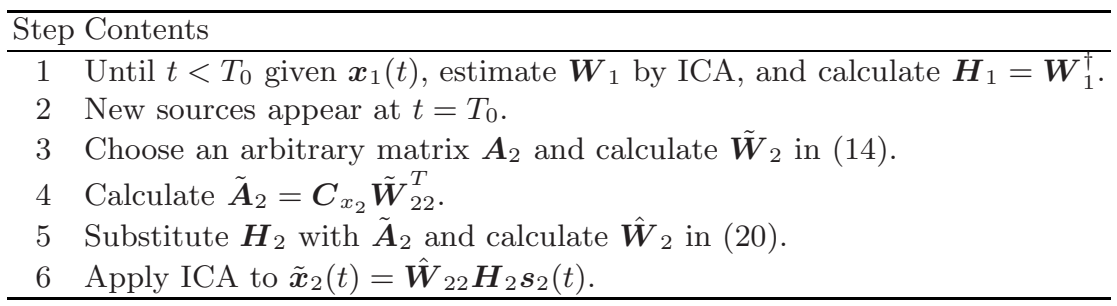

Since $\hat{\boldsymbol{W}}_{2}$ has the same property as $\tilde{\boldsymbol{W}}_{2}, \hat{\boldsymbol{W}}_{21}$ and $\hat{\boldsymbol{W}}_{22}$ work as blockers of $\boldsymbol{H}_{2}$ and $\boldsymbol{H}_{1}$, respectively, so that the product of $\hat{\boldsymbol{W}}_{2}$ and $\boldsymbol{x}_{2}(t)$ is calculated as follows:

$$
\begin{aligned}
\hat{\boldsymbol{W}}_{2} \boldsymbol{x}_{2}(t) & =\left[\begin{array}{ll}
\hat{\boldsymbol{W}}_{21} \boldsymbol{H}_{1} & \hat{\boldsymbol{W}}_{21} \boldsymbol{H}_{2} \\
\hat{\boldsymbol{W}}_{22} \boldsymbol{H}_{1} & \hat{\boldsymbol{W}}_{22} \boldsymbol{H}_{2}
\end{array}\right]\left[\begin{array}{l}
\boldsymbol{s}_{1}(t) \\
\boldsymbol{s}_{2}(t)
\end{array}\right] \\
& =\left[\begin{array}{cc}
\boldsymbol{I}_{n_{1}} & \boldsymbol{O}_{n_{1} \times n_{2}} \\
\boldsymbol{O}_{n_{2} \times n_{1}} & \hat{\boldsymbol{W}}_{22} \boldsymbol{H}_{2}
\end{array}\right]\left[\begin{array}{l}
\boldsymbol{s}_{1}(t) \\
\boldsymbol{s}_{2}(t)
\end{array}\right] \\
& =\left[\begin{array}{c}
\boldsymbol{s}_{1}(t) \\
\tilde{\boldsymbol{x}}_{2}(t)
\end{array}\right] .
\end{aligned}
$$

As a result, the BSS problem of $\tilde{\boldsymbol{x}}_{2}(t)=\hat{\boldsymbol{W}}_{22} \boldsymbol{H}_{2} \boldsymbol{s}_{2}(t)$ has only to be solved. Our method avoids to calculate the whole separating matrix $\boldsymbol{W}_{2}$ by ICA. It implies a low computational cost. The proposed method is summarized in Table 1.

\section{Simulation}

For the sake of clarity of the proposed method, BSS computational simulations were conducted on a PC (Core2 Duo E4300, 1.0GB RAM) using FastICA algorithm 9] implemented on MATLAB. Speech signals were used as source signals. The number of initial sources $n_{1}$ was four and the number of additional sources $n_{2}$ was varied from one to three. The number of sensors $m$ was seven and the mixed signals were generated through a mixing matrix. The coefficients of the mixing matrix were set randomly. We measured the processing time and the separation performance in order to evaluate the proposed method and the conventional method, which estimates a new separating matrix without any initialization. As a performance measure, signal to interference ratio (SIR) was calculated as follows:

$$
\mathrm{SIR}=\frac{1}{n_{1}+n_{2}} \sum_{i=1}^{n_{1}+n_{2}} 10 \log _{10} \frac{\sum_{t} y_{i, s_{j}}^{2}(t)}{\sum_{k \neq j} \sum_{t} y_{i, s_{k}}^{2}(t)},
$$

where $y_{i, s_{j}}(t)$ and $y_{i, s_{k}}(t)$ are $i$ th separated signals which are contributed by a certain source $s_{j}(t)$ and an interfering source $s_{k}(t)$, respectively. The results of 100 trials are shown. Fig. 3 (a) illustrates the processing time varying the number of the new sources. It is clear that the processing time using the proposed 


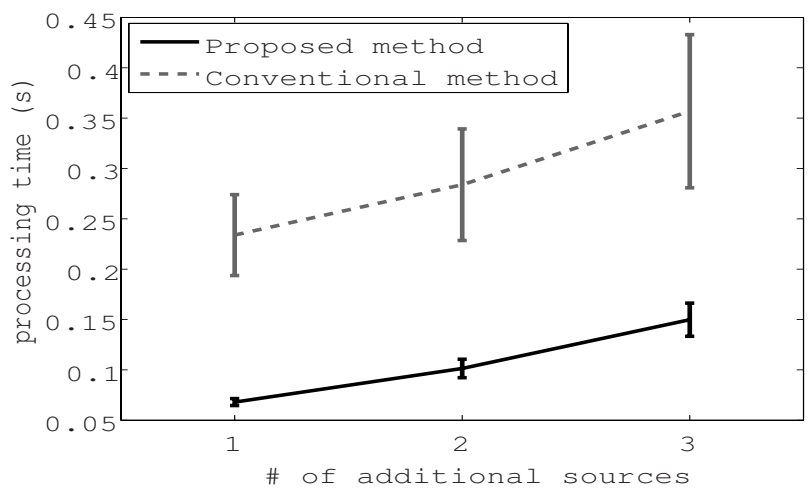

(a)

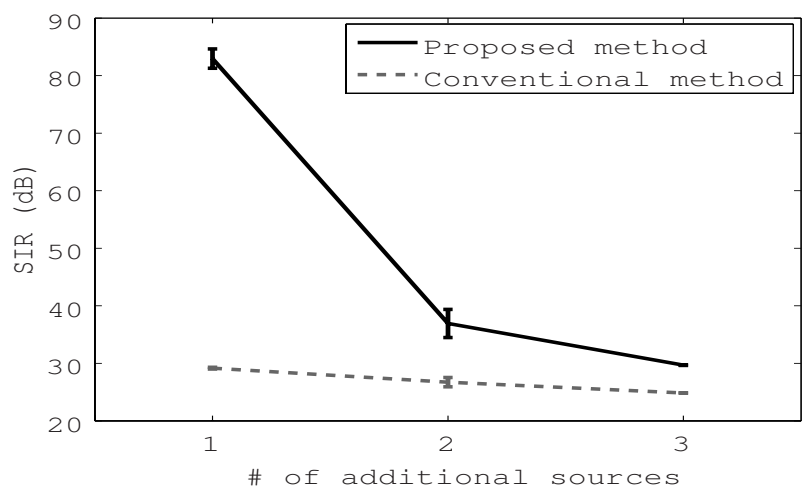

(b)

Fig. 3. The performance of the proposed method and the conventional method varying the number of additional sources. (a) The averaged processing time with standard deviation. (b) The averaged SIR with standard deviation.

method is significantly smaller than the conventional one. Our concern lies in the fact to estimate as $n_{2} \times n_{2}$ separating matrix, instead of the conventional method that estimates $\left(n_{1}+n_{2}\right) \times m$ separating matrix. Fig. 3 (b) depicts the separation performance. It is shown that our method outperforms the conventional method since the blockers work efficiently. Furthermore the conventional method caused permutation problems due to re-estimation of the separating matrix. The output channels of initial sources were different from those obtained by initial matrix, while using the proposed method the initial sources $\boldsymbol{s}_{1}(t)$ are given in the same order despite the appearance of new sources.

\section{Discussion}

The problem of detecting the number of active sources is very important for BSS and have been discussed by many authors 101112. Although some problems 
need to be solved, for instance when we have additional sources that appear after estimation of the separating matrix. In this research we proposed a new method that is based on the number of souces known a priori and the number of the dominant eigenvalues of the covariance matrix that can be solved straightforward using the well known FastICA algorithm. The highlight in this problem is to determine the sources dimension that is not solved easily by any BSS algorithm.

Until now we discussed the separation in the case when new sources appear. In addidion, the separation with a disappearance can be solved by a simple procedure based on the columns of the mixing matrix. Moreover, if a disappearance is detected the output energy become zero, which make the algorithm identify the vanished sources. Consequently, the corresponding columns of the vanished sources are removed from the mixing matrix, which was estimated before the disappearance, resulting in a new mixing matrix. Finally a new separating matrix is given as a pseudo inverse of the mixing matrix.

\section{Conclusion and Future Works}

In this manuscript we have proposed a scheme to separate mixed signals in the situation of new sources appearing. Utilizing the covariance matrix of the mixed signals, blockers of the initial sources and the additional sources can be obtained. As a result, blind separation of the new sources is only needed. Thus the computational load can be reduced compared with the conventional method. Through a computer simulation validity of the proposed method is shown.

Assuming that the matrix $\boldsymbol{M}=\boldsymbol{C}_{s_{2}} \boldsymbol{H}_{2}^{T} \tilde{\boldsymbol{W}}_{22}^{T}$ is nonsingular, namely $\tilde{\boldsymbol{W}}_{22} \boldsymbol{H}_{2}$ is also regular. To obtain $\tilde{\boldsymbol{W}}_{22}$ an arbitrary matrix $\boldsymbol{A}_{2}$ is used, further investigation about the way of choosing $\boldsymbol{A}_{2}$ is needed such that $\tilde{\boldsymbol{W}}_{22} \boldsymbol{H}_{2}=\left\{\boldsymbol{P}_{1} \boldsymbol{A}_{2}\right\}^{\dagger} \boldsymbol{H}_{2}$ is full rank.

The next goal is the extension of the proposed method to convolutive mixture to achieve blind separation of audio signals.

\section{References}

1. Hyvärinen, A., Karhunen, J., Oja, E.: Independent Component Analysis. John Wiley \& Sons, Inc., Chichester (2001)

2. Lee, T.W.: Independent Component Analysis. Kluwer Academic Publishers, Dordrecht (1998)

3. Ye, J.M., Zhu, X.L., Zhang, X.D.: Adaptive blind separation with an unknown number of sources. Neural Computation 16, 1641-1660 (2004)

4. Ito, M., Takeuchi, Y., Matsumoto, T., Kudo, H., Kawamoto, M., Mukai, T., Ohnishi, N.: Moving-source separation using directional microphones. In: Proceedings of the 2nd International Symposium on Signal Processing and Information Technology, pp. 523-526 (2002)

5. Mukai, R., Sawada, H., Araki, S., Makino, S.: Blind source separation for moving speech signals using blockwise ica and residual crosstalk subtraction. IEICE Trans. Fundamentals E87-A(8), 1941-1948 (2004)

6. Golub, G.H., Van Loan, C.F.: Matrix Computaions, 3rd edn. Johns Hopkins (1996) 
7. Inouye, Y., Liu, R.W.: A system-theoretic foundation for blind equalization of an fir mimo channel system. IEEE Trans. Circuits and Systems I: Fundamental Theory and Applications 49(4), 425-436 (2002)

8. Rao, C.R., Mitra, S.K.: Generalized Inverse of Matrices and its Applications. John Wiley \& Sons, Chichester (1971)

9. Hyvärinen, A.: Fast and robust fixed-point algorithms for independent component analysis. IEEE Trans. on Neural Networks 10(3), 626-634 (1999)

10. Wax, M., Kailath, T.: Detection of signals by information theoretic criteria. IEEE Trans. Acoustics, Speech and Signal Processing 33, 387-392 (1985)

11. Sawada, H., Mukai, R., Araki, S., Makino, S.: Estimating the number of sources using independent component analysis. Acoustical Science and Technology 26(5), 450-452 (2005)

12. Olsson, R.K., Hansen, L.K.: Estimating the number of sources in a noisy convolutive mixture using bic. In: Proceedings of the 5th International Conference on Independent Component Analysis and Blind Signal Separation, pp. 618-625 (2004) 